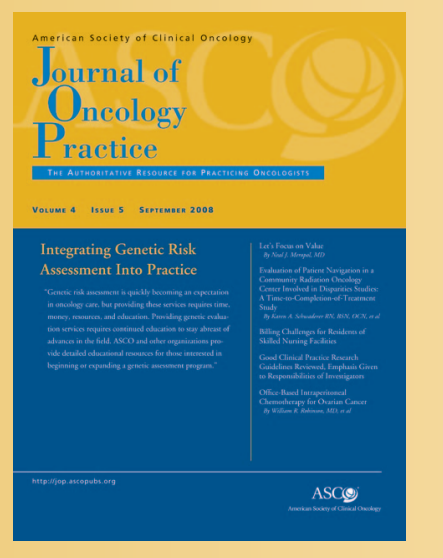

\title{
Integrating Genetic Risk Assessment
} Into Practice

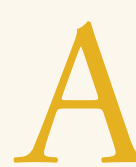

$s$ the research in clinical cancer genetics advances, offering risk assessment is becoming more of an expectation in oncology practice. When considering this service in a community oncology practice, it is important to realize the demands involved. A genetic risk assessment program requires a significant time and financial commitment that all practice members must be willing to fully embrace. When choosing to pursue a genetic program, a practice needs to have properly trained professionals available who have some understanding of cancer genetics, can adequately conduct a family history and interpret risk, and be able to provide counseling and interpret test results. This article provides more detail on the aspects of a genetic risk assessment program practicing oncologists need to consider, including:

- Adequate understanding of cancer genetics and hereditary syndromes

- Family history

- Risk assessment

- Counseling

- Testing

- Follow-up

- Billing

This is a general overview. If you are interested in beginning a risk assessment program or expanding your current risk assessment services, you should delve into understanding each of these topics and identify what will work best within your own practice.

\section{Cancer Genetics}

Hereditary cancer syndromes account for approximately 5\% to $10 \%$ of all cancers. That means about 50,000 newly diagnosed cancers each year in the United States are associated with altered susceptibility genes. ${ }^{1}$ Those patients are often at risk of recurrent or second cancers and in many instances, they have family members at risk of developing cancer because of the same mutation.

All cancers develop from gene mutations, but some people are born with a DNA mutation that predisposes them to disease.
Not everyone with a specific mutation will develop cancer, but their risk is greatly increased. Many people with an inherited mutation develop cancer at younger ages than the rest of the population.

Many of the mutations that lead to cancer follow autosomal dominant inheritance. In this case, the mutation happens in one copy of the gene, giving the carrier a $50 \%$ chance of passing it on. With autosomal inheritance both males and females have an equal chance of carrying the mutation.

Dominant inheritance means that only one copy of the allele is needed for the trait to be expressed. Because most mutations are not completely penetrant, the cancer may not affect all carriers in a family. When reviewing a family history it may look like the cancer skipped a generation, when the mutation was actually still being passed down. ${ }^{1}$

The main factors signaling an inherited cancer risk include:

- Clustering of the same type of cancer in close relatives

- Unusually early age of onset

- Two or more primary cancers in an individual

- Bilateral cancer in paired organs

- Multifocal cancer

- Patterns of cancer in the family that are associated with a known cancer syndrome

Table 1 lists common hereditary syndromes, the commonly occurring cancers, and the genes associated with each. Testing is available for mutations in each gene listed. Many cancer syndromes have red flags specific to them. Warning signs for each syndrome can be found on ASCO's Web site, www.asco. org The National Cancer Institute's Web site, www.cancer. gov and the National Comprehensive Cancer Network Web site, www.nccn.org.

Human genetics is a rapidly growing field, with new information constantly being uncovered. Staying abreast of this information requires a large time commitment. Successful programs employ nurses or genetic counselors who have received specialized training and are devoted to staying current in cancer genetics. This helps ensure that patients are receiving the best care possible. 
Table 1. Hereditary Syndromes Leading to Cancer

\begin{tabular}{|c|c|c|}
\hline Syndrome & Cancer & Gene \\
\hline Retinoblastoma & Retinoblastoma* & $R B 1$ \\
\hline MEN2A and MEN2B & Medullary thyroid & $R E T$ \\
\hline Li-Fraumeni syndrome & $\begin{array}{l}\text { Sarcomas, breast, brain, leukemia, adrenal cortical, } \\
\text { melanoma }\end{array}$ & TP53 (or p53) \\
\hline Hereditary breast and ovarian cancer & Breast, ovarian, prostate & BRCA1, BRCA2 \\
\hline FAP & $\begin{array}{l}\text { Colorectal, hepatoblastoma, papillary thyroid, } \\
\text { pancreatic, bile duct, adrenal }\end{array}$ & $A P C$ \\
\hline MYH-associated polyposis & Colorectal & $M Y H$ \\
\hline Hereditary melanoma/FAMMM & Melanoma & CDKN2A† (p16, MST1) \\
\hline HNPCC (Lynch syndrome) & Colorectal, endometrial, ovarian & MLH1, MSH2, MSH6 \\
\hline Cowden syndrome & Thyroid, breast, uterine & PTEN \\
\hline Peutz-Jeghers syndrome & Ovarian, breast, uterine, lung, colorectal, pancreatic & STK11 \\
\hline Juvenile polyposis syndrome & Colorectal, cancer in other areas of the digestive system & MADH4, BMPR1A \\
\hline Von Hippel-Lindau syndrome & Kidney & VHL \\
\hline HPRCC & Kidney & C-MET \\
\hline Birt-Hogg Dube syndrome & Kidney & $B H D$ \\
\hline HLRC & Kidney & $\mathrm{FH}$ \\
\hline Ataxia telangiectasia & $\begin{array}{l}\text { Leukemia, lymphoma, melanoma, sarcoma, breast, } \\
\text { ovarian, stomach }\end{array}$ & ATM \\
\hline Wilms tumor & Wilms tumor & WT1 \\
\hline
\end{tabular}

Abbreviations: MEN, multiple endocrine neoplasia; FAP, familial adenomatous polyposis; FAMMM, familial atypical multiple mole melanoma; HNPCC, hereditary nonpolyposis colorectal cancer; HPRC, hereditary papillary renal (cell) carcinoma; HLRC, hereditary leiomyomatosis and renal (cell) carcinoma.

* Children with hereditary retinoblastoma have an increased risk of developing osteosarcoma, other sarcomas, leukemia, lymphoma, melanoma, lung cancer, and bladder cancer as they age.

† Testing for CDKN2A is currently only recommended in a research setting.

\section{Family History}

Family history is the foundation for risk assessment and the basis for identifying patients at increased risk. Even when not providing full genetic assessment and testing services, oncologists are in a position to identify patients who may be at increased risk of cancer by recognizing the signs of an inherited syndrome. The general recommendation is to get a three-generational family history from all patients.

"It's incumbent on all oncologists to come up with a methodology for their practice to get a three-generational

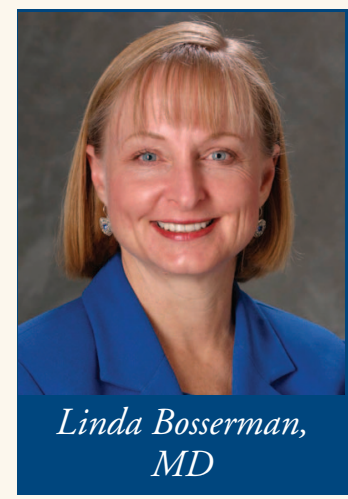
family history on each patient," said Linda Bosserman, MD, of Wilshire Oncology Medical Group, Inc (Pomona, CA). "This can be done through Web sites you link a patient to or through a paper intake."

This history includes information on first-, second-, and thirddegree relatives. Ask patients the type of each primary cancer, age at diagnosis, age at death, cause of death, and environmental exposures (smoking, radiation, and so on) of all relatives with cancer. Many patients may not know these details on all family members at their first visit, so it is important to regularly update the history. To verify the history, patients can take medical release consent forms to give to family members. Tumor registries and death certificates can also add information.

Oncologists are expected to understand genetic relationships and to ask detailed questions of patients, especially when patients may consider dramatic risk-reducing treatments based on family history. "Approximately $20 \%$ of the time, when a patient says there's no history, there really is a significant history because patients don't understand genetic relationship, and they don't realize how first and second cousins may be related," Dr Bosserman said.

\section{Risk Assessment and Counseling}

After reviewing a family history, there may be no indication of a hereditary syndrome, but it is best to update the family history at every visit in case a relative develops cancer. If, however, it seems that a hereditary syndrome may be present, it is important to discuss that risk with patients. That often leads to more detailed counseling. 
Counseling can be performed by genetic counselors or by other staff such as nurses who have received advanced training. Specifics of genetic counseling have been covered in previous Journal of Oncology Practice articles and in ASCO's policy statement on genetic cancer. ${ }^{2,3}$ In general, counseling educates patients about their cancer risk and about testing. Patients should be well informed of how test results are interpreted and that there is a risk of an inconclusive result. In addition, patients need to fully understand implications for the rest of their family, and the counselor should find out how a patient would feel about sharing the results with potentially affected family members. Counseling is a necessary step in the process and is critical to helping patients make informed decisions about testing.

Counseling before testing also includes obtaining informed consent from patients. Elements of informed consent include:

- Information on the specific test being performed

- Implications of positive and negative results

- Possibility that the test will not be informative

- Options for risk estimation without genetic testing

- Risk of passing a mutation to children

- Technical accuracy of the test

- Fees involved in testing and counseling

- Psychological implications of test results (benefits and risks)

- Risks of insurance or employer discrimination

- Confidentiality issues

- Options and limitations of medical surveillance and strategies for prevention after testing

- Importance of sharing genetic test results with at-risk relatives ${ }^{3}$

Counseling should be offered regardless of whether a patient chooses to undergo testing, and oncologists need to respect the patient's decision. "Some don't want to undergo testing, or are overwhelmed at first," Dr Bosserman said. "We work with them over time, and sometimes they come back months or even a year or two later and decide to have the test. There are a lot of emotional issues to work through leading up to testing and after, and oncologists should not be afraid of this.”

ASCO recommends offering counseling after the test as well. First, make sure patients want to know the results before disclosing them. In some cases, patients may decide they do not want to know yet. In general, post-test counseling includes interpreting the results for patients, fully explaining the implications of positive, negative, and inconclusive results; discussing implications for family members, offering patients guidance on how to share the results with family members, and discussing medical management options. During post-test counseling, providers can discuss available clinical trials for high-risk patients.

\section{Testing}

ASCO recommends only offering genetic testing when preand post-test counseling is available. If a patient wants to undergo testing, oncologists need to make sure they are using a qualified laboratory to perform the test. In addition, the best person in the family to test is a person who already has cancer, which can be a relative of the patient.

A variety of testing methods exist based on the type of mutation suspected. Testing for unknown mutations uses a wide array of molecular analytic techniques, and accuracy varies. Tests that search for a specific mutation can be suggested when there is reason to suspect it in a family or when a mutation is known to exist in other family members. The costs are reduced if a gene does not need to be sequenced completely.

Sequencing is the most commonly used method for detecting mutations related to cancer predisposition syndromes, but it is important to know what techniques the laboratory uses for mutation detection in order to interpret the results for a patient. Although gene sequencing is often informative, other techniques that look for gene rearrangements or deletions are also valuable and can be especially helpful when sequencing does not identify a mutation in a high-risk family. Sequencing is sometimes unable to distinguish between benign polymorphisms and disease-causing mutations.

When offering genetic testing, it is important to use a laboratory that adheres to high standards. Federal regulations are lacking, and the Clinical Laboratory Improvement Act of 1988 regulations are insufficient with today's technology. At a minimum, however, a laboratory should have Clinical Laboratory Improvement Act certification. Some professional societies also conduct inspection programs that laboratories can voluntarily participate in. A comprehensive list of laboratories that provides clinical genetic testing for all known cancer syndromes can be found at http://www.geneclinics.org/.

Laboratories should also provide technical support to help determine the best test for a patient, help navigate billing and insurance requirements, and provide detailed summaries of the test results to aid the patient discussion. For example, Dr Bosserman uses Myriad Genetics for testing, which provides educational information that she can provide to patients about testing and consultation with a geneticist or genetic counselor who can help determine the best options for her patients. In this case, she sends a patient's history and informed consent to the laboratory, the patient receives a test kit to take to a laboratory for blood work, which then gets sent to Myriad. Other laboratories, such as the Mayo Molecular Genetics Laboratory, also offer support from board-certified genetic counselors to assist with the interpretation of genetic test results. 


\section{Billing}

After deciding to offer genetic risk assessment, navigating the reimbursement process is another challenge. The reality is that reimbursement is usually not commensurate with the time spent providing care. In addition, reimbursement for testing has not kept up with the technology. But by understanding billing codes, bills can be submitted in the most effective way possible.

The most common billing codes used are the current procedural terminology (CPT) and evaluation and management (E\&M) codes. Tests are billed using pathology and laboratory codes, which identify the procedure being performed, not necessarily the test itself. A new test that performs the same procedure does not create a new code, so reimbursement rates may be based on an older method of performing the test. In addition, most health plans use the Medicare clinical laboratory fee schedule as a guide for their payment levels. In 2003, Medicare placed a fee freeze on these rates, locking in the 2003 rates until 2009. Consequently, these Medicare fees have not kept up with changes in technology or inflation and do not reflect the actual cost of many tests.

Genetic counseling services are billed using E\&M codes. There are some new codes introduced in CPT in 2007 for genetic counseling services for a 30-minute face-to-face visit with a patient or family. This code is intended to be used by genetic counselors who previously could not bill on their own. Medicare does not recognize this code as separately payable, but some private payors may. Physicians may bill for genetic counseling services provided by a genetic counselor as incident to the physician visit, as long as the physician is providing direct supervision. Physicians providing genetic counseling services themselves bill using the appropriate level E\&M code.

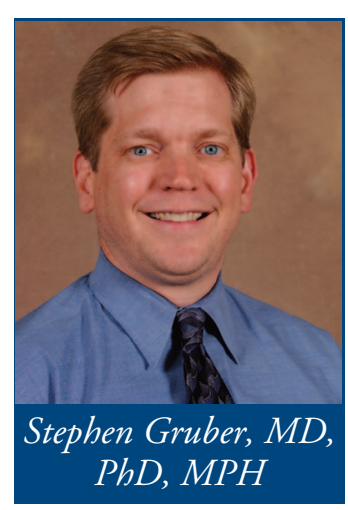

Though it seems inadequate to bill for an 80-minute visit when genetic counseling can take up to two hours, there are processes that fit within the billing system to provide care to patients. At the University of Michigan Cancer Genetics Clinic (Ann Arbor, MI), Stephen Gruber, $\mathrm{MD}, \mathrm{PhD}, \mathrm{MPH}$, spends approximately one hour with patients for an intake visit, which involves a physical examination, full patient/family

history, and discussion of management options in conjunction with a genetic counselor who provides risk assessment and counseling. This allows for billing using the E\&M code with counseling being incident to the physician visit. Even patients without a diagnosis of cancer, which comprise more than half of his patients, have a medical condition that warrants a genetic evaluation.

Dr Bosserman breaks up her visits into smaller sessions that can specifically address family history. Initial consultations include a three-generational risk assessment, but detailed discussions and counseling are often scheduled for separate appointments and may include other interested family members. Time is needed to address more detailed risk assessment, counseling, testing, and management of risk, whether or not patients choose testing. In addition, when patients with cancer come for their annual follow-up visits, it is an opportunity to update and review their family history. The risk of inherited cancer mutation can change with new family member diagnoses that might change the recommendations for genetic counseling or initiation of new risk reduction strategies.

"If you can break up the phases, it's less overwhelming to patients and physicians," Dr Bosserman said. She also advises that physicians judge how much to include in one visit by how much information their patients may be able to handle at once. "We know what level of detail our patients want. They don't often want a 3-hour genetic consult. Usually, they want things in perspective, just like we present the rest of complex cancer medicine," she said.

\section{Putting It All Together}

The staff and time involved in performing risk assessment can be intimidating to clinicians, but addressing risk is becoming more important as research discovers more about genetics. Many training courses and conferences about risk assessment programs are available, and elements of others' programs may be applied as well.

"Cancer genetic syndromes are typically under-recognized in the community, including the academic community," Dr Gruber said. "I think it's especially valuable for oncologists to be attentive to family history. Managing individuals at high risk makes a dramatic difference in lessening the risk of cancer in family members, and it's increasingly becoming the standard of care."

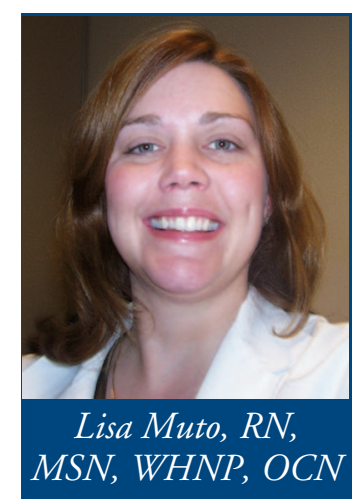

When considering offering or expanding any part of a genetic risk assessment program, additional education is a must. Lisa Muto, RN, MSN, WHNP, OCN, of Edwards Comprehensive Cancer Center (Huntington, WV), is beginning a risk assessment program at her practice. To begin, she researched programs online, joined professional societies such as the 
Oncology Nursing Society, and attended educational seminars. She recommends the Fox Chase Cancer Center's Basic Cancer Genetic Program for Nurses and City of Hope's Intensive Course in Cancer Risk Assessment.

"Every person I have met has taught me something that has helped me in some way," she said. "You need to take advantage of all the educational seminars or conferences you can because in the field of genetics, there is always new information."

After researching genetic counseling options, a practice should evaluate what is feasible and assess how it can provide these services. Multiple staff members may need to obtain additional training and it may become necessary to hire additional staff. Will the oncologists provide counseling? Should everyone or only someone with a special interest focus on this? Is a genetic counselor necessary, or can a nurse, nurse practitioner, or physician's assistant focus on genetics and offer counseling? Researching how other practices have addressed these questions may help answer them.

Genetic counselors are strongly recommended to provide counseling. "Genetic counselors, due to their extensive study of genetics, can help identify families or individuals who may be at increased risk for inherited conditions, especially those less common," said Kara Milliron, MS, CGC, genetics counselor at University of Michigan Health Systems (Ann Arbor, MI). However, not everyone is able to hire a genetic counselor. Some practices develop formal or informal partnerships between genetic counselors and busy oncologists. Another option, when seeing fewer patients, may be for the oncologist to provide counseling or have a staff member trained to do so. Ms Muto provides counseling at her clinic, while Dr Bosserman, her colleagues, and their nurse practitioner and physician's assistant partners are able to provide counseling for their patients.

Deciding to whom these services should be marketed is another consideration. Some offices offer genetic assessment and counseling to their regular patients only, whereas other offices have expanded services to the community, which brings in patients with a possible hereditary syndrome, but not a diagnosis of cancer. Dr Bosserman began offering risk assessment as a service to her patients and became known as a resource in her community. Some local primary care physicians refer patients with suspicious family histories for a cancer risk assessment. She evaluates these patients and recommends a follow-up plan for the primary physician to follow.

Many private practices focus primarily on patients with cancer. Ninety percent of Dr Bosserman's patients have breast cancer, and of about 350 patients a year and some follow-up patients where new family history increases the threshold for a positive test, about 25 to 30 patients receive testing. With those numbers, she is able to provide counseling herself and relies on the testing laboratory for support and tools to communicate results with patients. She has found that patients are grateful for this service and how it enhances their care.

"By offering genetic assessment, you become much more comprehensive, not just developing a treatment plan for the cancer in front of you, but also developing a long-term strategy for health and risk reduction for the patient and potentially for family members," Dr Bosserman said.

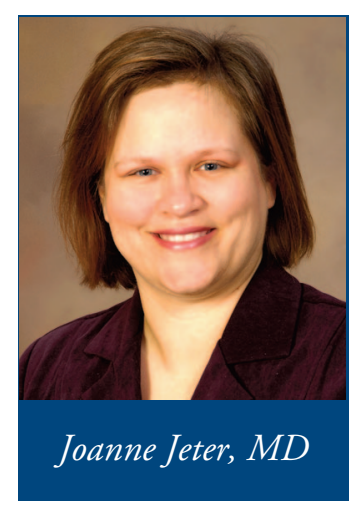

If the resources and patient volume are available, a more detailed clinic may be an option. Joanne Jeter, MD, of Arizona Cancer Center (Tucson, AZ), is developing a risk assessment clinic within the cancer center. Fortunately, she already had a genetic counselor on staff, but their goal was to build a clinic where patients could speak with multiple specialists on the same day including oncologists, a social worker and a dietician. The cancer center was used to receiving patients through referrals from within the system, but now its focus has shifted.

"When we started the risk assessment clinic, we had to start considering ourselves an entry point into the system, which was something of a paradigm shift," she said. "We had to educate many of our staff, including teleservices, billing, scheduling, and nursing on the different needs of these patients relative to those of oncology patients."

Dr Jeter also took a refresher course at City of Hope to become more familiar with genetics and to help establish connections with leaders in the field.

Sometimes partnering with an academic medical center in the area may be the best route. In this case, an oncologist still needs to obtain a family history and be able to recognize a potential hereditary syndrome. It is critical to know when to refer. At the Michigan clinic Dr Gruber heads, patients come through referrals from oncologists, surgeons, family practice physicians, and others in the community. He also holds outreach clinics in areas less likely to have access to these services. The clinic provides risk assessment counseling, testing, and medical advice, then sends patients back to their physicians for management. Another advantage to using a clinic such as the one at Michigan is that it provides community oncologists with access to tests and counselors for patients with less common hereditary syndromes.

Finally, having access to clinical trials can be a major asset to community oncologists. Patients with a less common hereditary syndrome may be best managed by a research 
study. Also, some genetic tests available are only offered in terms of research studies. Family members at high risk may also benefit from prevention studies.

Even if you take small steps into offering risk assessment, make sure to have a well-developed plan. With more and more being learned about cancer genetics, oncologists are expected to recognize patterns and provide more of these services, but providing them incorrectly opens up a physician to liability. ASCO's Cancer Genetics Curriculum serves as an

\section{References}

1. Offit K, Garber J, Grady M, et al: ASCO Curriculum: Cancer Genetics \& Cancer Predisposition Testing (ed 2). Alexandria, VA, American Society of Clinical Oncology, 2004

2. Genetic counseling: An Indispensable step in the genetic testing process. J Oncol Pract 4:96-98, 2008 excellent resource for understanding genetics, testing, and counseling. In addition, City of Hope offers a course to help physicians identify when to appropriately refer patients. By increasing your understanding of genetics, you'll be able to better manage your patients' medical condition and their future, possibly providing you with the opportunity for prevention.

DOI: 10.1200/JOP.0853501

3. American Society of Clinical Oncology Policy Statement Update: Genetic Testing for Cancer Susceptibility. J Clin Oncol 21:2397-2406, 2003

\section{APPLY NOW FOR THE CAREER DEVELOPMENT AWARD (CDA) \& THE YOUNG INVESTIGATOR AWARD (YIA)}

Over the past 25 years, The ASCO Cancer Foundation's Grants Program has awarded over $\$ 50$ million to deserving researchers. Online applications are currently being accepted for the Career Development Award, which provides funding to translational and clinical investigators in their second, third or fourth year of a faculty appointment to establish an independent clinical cancer research program and the Young Investigator Award, which provides funding to final year fellows or first year junior faculty, to encourage and promote quality research in clinical oncology. To apply now or for more information, visit www.ascocancerfoundation.org/grants.

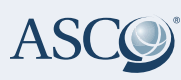

American Society of Clinical Oncology 